\title{
Importance Analysis of Civil Aircraft APU
}

\author{
Lixia Gao, Hanlin Shen \\ Civil Aviation Flight University of China, China \\ *gaolx0129@126.com
}

Keywords: high high-altitude; auxiliary function; fault monitoring; APU

\begin{abstract}
APU is an important part of the large and medium-sized passenger jets, which is used to provide auxiliary power for the aircraft, including the auxiliary gas source and the auxiliary power source. The auxiliary gas source supplied by APU can be used to engine starting or the environmental control system in emergency condition. The auxiliary power supply can be used to replace one or two fault main generators for the power supply in the case of one or two main generators failure. The civil aircraft running scale of high high-altitude is continuous growing. The operating environment of high high-altitude is much worse than the usual plain. In this paper, through the analysis of the auxiliary function of the APU, especially the importance of APU's word and the influence to the associated system in high high-altitude environments, the conclusive results can be achieved. On one side, it can not only help maintenance personnel to understand the importance of the APU deeply, to strengthen the significance in the process of maintenance, but also provide some reference for the relevant documents for the maintenance or operation of the high high-altitude.
\end{abstract}

\section{APU structures and functions}

APU is a small gas turbine engine APU essentially, including the compressor, combustor, turbine, ignition system, starting system and other components. As an auxiliary power unit of the aircraft, APU can supply auxiliary power source and air source for the aircrafts. It is a critical system to ensure safety and comfort of the aircraft.

The specific functions of APU includes: on the ground, in the absence of a suitable ground power and gas supply situation, if the APU start up, on the one hand, the APU engine produces gas source to start the main engine and supply gas for the environmental control system (ECS); on the other hand, the APU engine provide mechanical energy to the APU generator, the generator parameters consistent with the main generator, can supply power to the entire plane grid before the primary generator is started up and no external power source connected.APU is one of the important equipment used to startup the aircraft engine after air parking. Under the emergency conditions in the air, the auxiliary air supplied by APU can used to restart the stopped main engine or supply gas for the ECS, the auxiliary power source can be used to replace the failure main generator, to guarantee the power supply capacity of the power grid and important electrical equipment power supply redundancy. A small amount of APU can also provide additional thrust for the plane.

\section{APU start analysis}

The start machine for APU drives the APU engine starting. The start machine is supplied DC power from the aircraft network. Then the direct current is converted into mechanical energy to drive the APU engine.

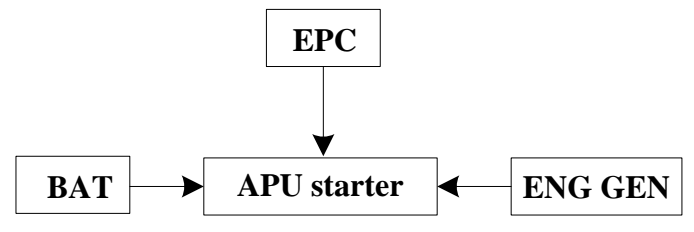

Figure1. APU starter power supply 
After APU start up, it can provide normal air source and power source. Usually, the DC power supplied to the APU start machine can come from the ground power conversion, onboard battery, and the normal work main generator. The APU specific power source constitution is shown in Fig.1.

In general, plain airports are equipped with suitable ground power, which can supply APU start machine. Most airports not only has ground power, but also equipped with a ground air source. Under this condition, APU doesn't need to start, because ground air source can directly be used to start the main engine. Using the ground air source to start the main engine can reduce the cost of operations.

Table I . Basic condition of the high high-altitude airport in China

\begin{tabular}{lcc}
\hline \multicolumn{1}{c}{ Airport name } & Airport navigation time & Altitude $(\mathrm{m})$ \\
\hline Lhasa Kongga International Airport & 1965 & 3600 \\
GeEr Mu Airport & 1974 & 2842 \\
QamdoBangda Airport & 1995 & 4334 \\
DiqingXianggelila Airport & 1999 & 3288 \\
JiuzhaiHuanglong Airport & 2003 & 3448 \\
Nyingchi Mainling Airport & 2007 & 2949 \\
Kangding Airport & 2007 & 4280 \\
Yushu Airport & 2009 & 3950 \\
NagriGunsa Airport & 2010 & 4274 \\
Shigatse Peace Airport & 2010 & 3782 \\
GannanXiahe Airport & 2013 & 3189 \\
Aden Daocheng Airport & 2013 & 4411 \\
ShenNongJia Airport & 2014 & 2580 \\
Aba Hongyuan Airport & 2014 & 3535 \\
NinglangLuguhu Airport & 2015 & 3300 \\
\hline
\end{tabular}

In china, the high high-altitude airport with the altitude is above 2438 meters (8000 feet) mainly concentrated in the southwest region. Now the number of the opened airport is at 15 , which accounts for about half the total number of the world's high high-altitude airport in service. The characteristics of the high high-altitude are complex geographical environment, poor clearance condition, the volatile weather, the uniqueness of the region, the weather phenomenon such as frequent wind, thunderstorms, low cloud and low visibility, wind shear, etc. The basic condition of the high high-altitude airport in China is shown in table I.

Due to the special geographical conditions and climatic conditions restrictions on high high-plateau region, to ensure the flight safety, the civil aviation administration made more stringent requirements for the protection of the safe operation of aircraft on high high-altitude airport.

Now some of the high high-altitude airports in operation are not equipped with suitable ground power and air supply, thus the gas is used to start main engine supplied by APU. If it is a short stop, generally APU is not required to stop. But if special circumstances leading to APU parking, it need to start APU firstly before the main engine starts. In this case, APU starting relies on on-board battery power. In the process of the APU start, the battery output voltage and output power are required to maintain above a certain value, in order to meet the input voltage and the starting power requirements of the power conversion unit.

The main models participate in the high high-plateaurunning are A320 and 737NG. The onboard batteries are nickel-cadmium batteries. For such batteries, the low-temperature discharge, high-current discharge, long time discharge will greatly reduce the battery life. And the low-temperature and high-current discharge will cause the battery voltage drops rapidly, to reach the discharge end voltage in a very short time. When the APU starts, long starting time also has an adverse effect on the APU. Thus, if the onboard battery is used to start the APU, to ensure the battery capacity enough is important, and the APU starting system must also be reliable. So the APU can reliably start up in a short period of time, in order to reduce the damage of battery and APU. 
According to the existing requirements related to the operation of high high-altitude, the APU of aircrafts running on high high-altitude airport should have capacity for independent startup in the running airport. Currently, not all high high-altitude airports have ground power and air supply to meet the requirements of the aircrafts, when to start the main engine in the high high-altitude airport, APU's successful start is crucial.

Combining these two points above, the current common practice in the flight manual is when close to the high high-altitude airport, to start the APU when the height is low than 20,000 feet. If the APU does not start, the pilot can choose the return. If the air APU start is successful, on the one hand, to prepare for possible overshoot. On the other hand, when the aircraft will have a short shop on the ground, if the airport does not have a suitable ground power or gas source provided, the APU can be used to provide a gas source to start the engine, and to provide power to the service electricity supply and maintenance electricity supply.

\section{Analysis of APU gas auxiliary function}

\subsection{Normal operating environment}

During the normal operation, the aircraft engine thrust can meet the requirements of the aircraft, which can guarantee the supply of the aircraft gas source, restart one shutdown engine, provide sufficient thrust when the plane needs to overshoot.

\subsection{High high-altitude operating environment}

When the aircraft runs at high high-altitude environment, it will be influenced by the environmental and climatic conditions, then lead to some features of the key system are affected, such as brake systems, engine systems. Especially in aircraft taking off and landing phase, the engine thrust influenced by the environmental will be reduced. In this case, if the special situation occurs in decline stage, the aircraft needs to overshoot, in order to ensure enough thrust for engine to climbing or obstacle, it need the main output power of the engine to create the climbing thrust, so the gas source needs inside the aircraft is supplied by APU. Therefore, APU is needed to operate correctly in the high high-altitude airport.

In addition, the air density of the high high-altitude airport is small. The effective thrust of the engine is reduced accordingly. If the icing phenomenon occurs in the aircraft taking off and landing stage, to reduce icing hazards to the flight, engine bleed air is needed to anti-icing, this will lead to thrust further reduced. If the aircraft air-conditioning systems also use engine bleed air, it will lead to a more part loss of the engine thrust, this will greatly increase the risk of overshoot. In order to ensure the reliability and security of the climb or obstacle may appear, APU is selected to supply air source for the air conditioning system, which can effectively reduce the use of engine thrust, improving the reliability of the aircraft overshoot.

\section{Analysis of APU power auxiliary function}

\subsection{Normal operating environment}

During the normal operation, the engine thrust meets the primary requirements of the aircraft. It can safeguard the sufficient mechanical energy for the main generator. So it can create the constant frequency AC power meeting the required parameters. The power supply has high reliability itself, and thus the reliability of the power supply system is guaranteed. When APU generator is working properly, it is a very important source of the aircraft power supply system. When one or more main generators are not working, APU generator replaces the failure main generator.

\subsection{High high-altitude operating environment}

During the high high-altitude operation, due to the impact of high altitude special environmental conditions, the possibility of single engine failure during aircraft taking off and landing is increasing, which will lead to the failure of the main engine-driven generator. The aircraft can no longer power grid, causing the aircraft the 
number of available grid power supply to reduce, reducing the supply of aircraft electrical system and the power supply redundancy of the important bus bar. If the power supply redundancy of critical electrical equipment is reduced, then this will reduce the reliability of equipment and systems, thereby endangering the safe operation of aircraft. The APU generator is used to replace the main failure power supply to ensure the requirements of power supply reliability for the supplied equipment and the corresponding system.

\section{APU fault monitoring}

If an emergency happens, leading to the APU system to stop APU. When on ground, if the APU is on fire and an overtemperature at APU compartment is found by overheat detection system, the system is automatically activated. At this time, the APU can be stopped manually through the cockpit or the external power supply control panel which is front the landing gear.

The monitoring of APU mainly includes speed monitoring, exhaust gas temperature (EGT) monitoring, the amount of oil monitoring and other signals, to confirm whether the APU is operating normally. If APU is in failure, it will not be able to perform a supporting role. For high high-altitude operation, the APU fault is discovered before takeoff, the plane will not be released. If the discovery happens during the running, the plane is to return in time.

\section{Conclusions}

Through the above analysis of the importance of the APU, and in particular, the importance of the special flight environment analysis, it is clear that, to protect the safety of flight operations, the reliable work of APU is vital. In order to ensure reliability in the operation of the APU, it needs aircraft maintenance personnel are fully aware of the problems, give more attention about it in routine maintenance. To achieve the purpose, the double standards can be adopted in the APU system maintenance.

\section{References}

[1] Li Li2014 Experimental study APU cooling system flying transport aircraft Aeronautical Science and Technology

[2] Jianliang Mao 2014 APU troubleshooting experience at high altitude airport operating conditions Aviation Maintenance \& Engineering

[3] LeYang2007 critical technology researchof the civilian aircraft takeoff performance on high altitude airports (Nanjin: Nanjing University of Aeronautics and Astronautics )

[4] China Eastern Airlines CoLtdYunnan 2011 B737-700 / 800 MEL

[5] Air China 2011 A320 family aircraft minimum equipment list

[6] Gyu-ho Kim, Bok-won Lee,Hongbing Lu2012 Failure analysis of an aircraft APU exhaust duct flange due to low cycle fatigue at high temperatures Engineering failure analysis 